\title{
Universal Scaling of Surface Plasmon Modes
}

\author{
F. P. Schmidt ${ }^{1,2}$, H. Ditlbacher ${ }^{1}$, U. Hohenester ${ }^{1}$, A. Hohenau ${ }^{1}$, F. Hofer ${ }^{2}$ and J. R. Krenn ${ }^{1}$ \\ ${ }^{1 .}$ Institute of Physics, University of Graz, Graz, Austria \\ 2. Institute for Electron Microscopy and Nanoanalysis (FELMI), Graz University of Technology, Graz, \\ Austria
}

Plasmons are collective electron oscillations, preferentially excitable in metals and can be seen as propagating density waves of conduction electrons. They are whether of bulk type and propagate within the material ("3D plasmon"), or along the metal's surface (2D or surface plasmon). In nanooptics surface plasmons are of special interest, because they can be excited optically (generated at optical frequencies) and show confinement into areas smaller than the wavelength of light, which causes a strong increase of the electric field intensities. This and the ability of surface plasmons to transform an optical signal into a quasi 2D state, also described as "flat light", make them a promising candidate for a multitude of applications.

Reducing the dimensions of metallic structures down to the nanometer regime, leads to a pronounced enhancement of the electromagnetic field intensities, when surface plasmons are generated. This fact can be understood, if metal nanostructures are seen as a kind of resonator for surface plasmons, which are reflected at the particle's edge, leading to standing wave like excitations (so-called localized surface plasmons). Various applications, ranging from novel light sources, photovoltaics to sensor devices, take advantage of the extraordinary optical properties of such metallic nanostructures.

Instead of light we used a monochromated $200 \mathrm{keV}$ electron beam in a scanning transmission electron microscope (FEI Tecnai F20) to probe the evanescent fields of localized surface plasmons with nanometer resolution. The electron energy-loss (EEL) was measured in order to get spatially resolved spectral information from the near-infrared, the visible and the UV range [1]. As the acquired signals are related to the plasmonic density of states, this method is a powerful complement to optical nanospectroscopy.

Nanodisks and straight (nano)edges of different size were designed using electron beam lithography (Figure 1). We show that the multitude of localized surface modes observed on these structures can be reduced to two main excitations. First we identify a radially symmetric ("breathing") dark mode. By tracing the dispersion of this mode for particles with different diameters we find that it corresponds to the extended two-dimensional surface plasmon, with a wavenumber determined by the disk diameter [2]. Second, we find that the dipolar and multipolar disk modes that show a dispersion, with wavenumbers set by the disk's circumference, that differ from the breathing mode (and 2D surface plasmon) dispersion but are very close to that of straight edges (Figure 2). These experimental observations together with a comprehensive theoretical study in the form of boundary element method (BEM) simulations [3] lead us to universal scaling rules, connecting the nanodisk spectrum to the surface plasmon modes of extended films and edges [4].

\section{References:}

[1] A.L. Koh et al, Nano Letters 11 (2011), 1323. 
[2] F.P. Schmidt et al, Nano Letters 11 (2012), 5780.

[3] U. Hohenester and A. Truegler, Comp. Phys. Commun. 183 (2012), 370.

[4] The authors gratefully acknowledge funding from the Austrian Science Fund under grant numbers P21800-N20 and P24511-N26, ESTEEM2 (FP7 project, No.312483), SFB F49 NextLite and the Graz Center for Electron Microscopy.

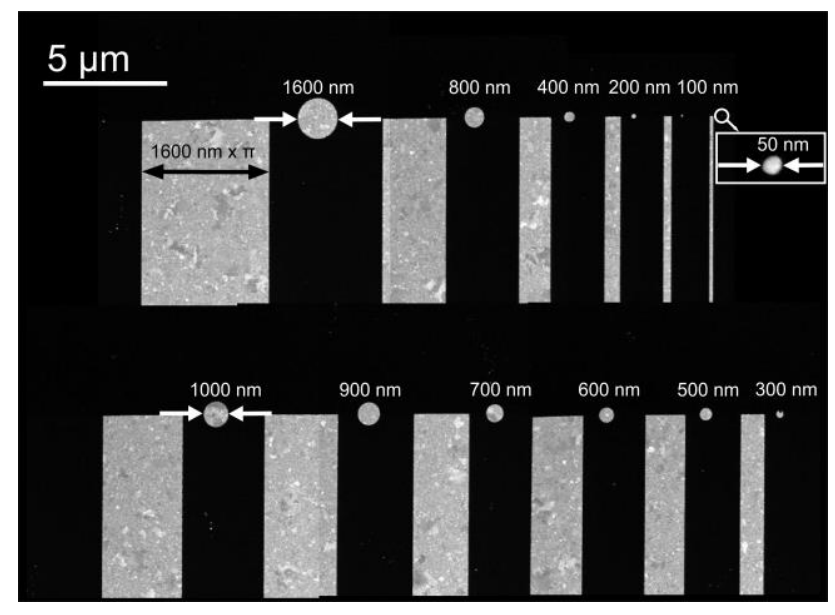

Figure 1. Lithographed silver disks and rectangles of $30 \mathrm{~nm}$ thickness on a $15 \mathrm{~nm}$ thin $\mathrm{Si}_{3} \mathrm{~N}_{4}$ membrane. The width of the rectangle is equal to the disk's circumference, where the diameter is ranging from 1.6 $\mu \mathrm{m}$ to $50 \mathrm{~nm}$. The orthogonal direction of the rectangles was kept constant to $100 \mu \mathrm{m}$.
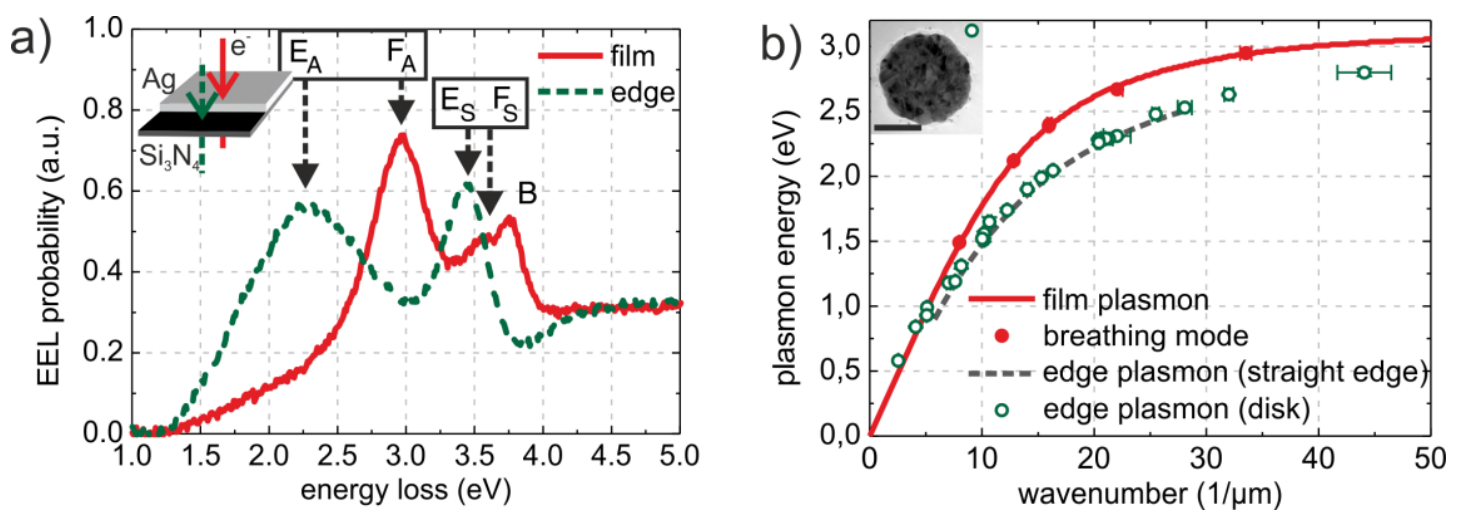

Figure 2. a) Extended silver film: Zero loss subtracted EEL spectra taken on a $30 \mathrm{~nm}$ thick silver film on a $15 \mathrm{~nm}$ thin $\mathrm{Si}_{3} \mathrm{~N}_{4}$ membrane (solid line) and outside the silver film, $15 \mathrm{~nm}$ beside the edge (dashed line) as indicated by the inset in the upper left corner. b) Surface plasmon dispersion of an extended $\mathrm{Ag} / \mathrm{Si}_{3} \mathrm{~N}_{4}$ system (solid line) and of disk breathing modes (filled circles) are compared to edge mode dispersion of disks (unfilled circles) and straight edges (dashed line). Silver thickness and Si3N4 thickness are the same as in a), the scale bar in the inset is $100 \mathrm{~nm}$. 